SPSS for Social Scientists 


\section{SPSS for Social Scientists}

Robert L. Miller, Ciaran Acton, Deirdre A. Fullerton and John Maltby

Consultant editor: Jo Campling 
All rights reserved. No reproduction, copy or transmission of this publication may be made without written permission.

No paragraph of this publication may be reproduced, copied or transmitted save with written permission or in accordance with the provisions of the Copyright, Designs and Patents Act 1988, or under the terms of any licence permitting limited copying issued by the Copyright Licensing Agency,

90 Tottenham Court Road, London W1T 4LP.

Any person who does any unauthorised act in relation to this publication may be liable to criminal prosecution and civil claims for damages.

The authors have asserted their rights to be identified as the authors of this work in accordance with the Copyright, Designs and Patents Act 1988

First published 2002 by

PALGRAVE MACMILLAN

Houndmills, Basingstoke, Hampshire RG21 6XS and

175 Fifth Avenue, New York, N.Y. 10010

Companies and representatives throughout the world

PALGRAVE MACMILLAN is the global academic imprint of the Palgrave Macmillan division of St. Martin's Press, LLC and of Palgrave Macmillan Ltd. Macmillan ${ }^{\circledR}$ is a registered trademark in the United States, United Kingdom and other countries. Palgrave is a registered trademark in the European Union and other countries.

ISBN 978-0-333-92286-6 ISBN 978-0-230-62968-4 (eBook) DOI $10.1007 / 978-0-230-62968-4$

This book is printed on paper suitable for recycling and made from fully managed and sustained forest sources.

A catalogue record for this book is available from the British Library.

A catalogue record for this book is available from the Library of Congress.

$\begin{array}{rrrrrrrrrr}10 & 9 & 8 & 7 & 6 & 5 & 4 & 3 & 2 & 1 \\ 11 & 10 & 09 & 08 & 07 & 06 & 05 & 04 & 03 & 02\end{array}$

Typeset in Great Britain by Aarontype Ltd, Easton, Bristol 


\section{CONTENTS}

List of Figures $\quad$ x

Preface $\quad$ xv

Acknowledgements $\quad$ xvii

Introduction $\quad 1$

The schism between quantitative and qualitative perspectives 1

Two quantitative perspectives

The empirical $\quad 2$

The positivist 3

$\begin{array}{ll}\text { Orientation } & 10\end{array}$

The British Social Attitudes Survey 10

The datasets $\quad 10$

Obtaining the practice datasets $\quad 11$

Introduction to the workbook

Introduction to SPSS for Windows $\quad 12$

$\begin{array}{ll}\text { The Online Tutorial } & 12\end{array}$

The Statistics Coach $\quad 12$

The Contextual Help System $\quad 12$

Getting started on SPSS 12

(1) The Title bar 13

(2) The Menu bar $\quad 15$

(3) The Tool bar 16

Some minor adjustments to SPSS 16

$\begin{array}{ll}\text { Resetting variable lists } & 17\end{array}$

Resetting output labels $\quad 17$

Loading a data file $\quad 18$

The Viewer window $\quad 22$

Saving to a disk $\quad 25$

Saving the data file $\quad 25$

Saving your output file $\quad 27$

$\begin{array}{ll}\text { Printing your SPSS output } & 28\end{array}$

1 Data Input $\quad 30$

Cases and variables $\quad 30$

'Rectangular' format 31

Exercise $\quad 32$ 
Inputting data into SPSS

Option 1: importing an SPSS portable file $\quad 32$

Option 2: importing data from spreadsheets $\quad 34$

Option 3: importing text files $\quad 35$

Option 4: creating a new SPSS data file $\quad 36$

$\begin{array}{ll}\text { Editing data } & 38\end{array}$

$\begin{array}{ll}\text { Entering new variables or cases } & 38\end{array}$

Deleting a variable or a case $\quad 38$

Saving new files $\quad 39$

Labelling variables $\quad 40$

Coding $\quad 41$

Quantitative data $\quad 41$

Qualitative data $\quad 42$

'String' data $\quad 43$

Refining the dataset $\quad 43$

Labelling the data $\quad 44$

SPSS operations to 'label' and 'refine' a dataset $\quad 45$

Validating $\quad 49$

Dealing with missing values $\quad 51$

Conclusion $\quad 53$

Some tips

SPSS exercise $\quad 54$

Appendix 1: Student 'Drinking questionnaire'

Appendix 2: Labelling using SPSS V9 56

2 Listing and Exploring Data $\quad 59$

Introduction $\quad 59$

Levels of measurement $\quad 59$

Nominal/Categorical $\quad 59$

Ordinal $\quad 59$

Interval $\quad 60$

Ratio $\quad 60$

$\begin{array}{ll}\text { Frequency tables } & 61\end{array}$

$\begin{array}{ll}\text { Measures of central tendency } & 63\end{array}$

$\begin{array}{ll}\text { The mean } & 64\end{array}$

$\begin{array}{ll}\text { The median } & 64\end{array}$

$\begin{array}{ll}\text { The mode } & 65\end{array}$

Measures of dispersion $\quad 65$

$\begin{array}{ll}\text { The range } & 65\end{array}$

$\begin{array}{ll}\text { The interquartile range } & 65\end{array}$

$\begin{array}{ll}\text { The variance } & 65\end{array}$

The standard deviation $\quad 66$

Descriptive statistics and charts in SPSS $\quad 66$

Example I $\quad 66$

Example 2 69

$\begin{array}{ll}\text { Explore } & 72\end{array}$

$\begin{array}{ll}\text { Other graphs and charts } & 78\end{array}$

$\begin{array}{ll}\text { Pie charts } & 78\end{array}$

$\begin{array}{lr}\text { Scatterplots } & 80\end{array}$ 
Line charts

The Chart Editor

SPSS exercise for Module 2

3 Data Selection and Manipulation $\quad 88$

Introduction $\quad 88$

Data selection $\quad 88$

Subsets of cases $\quad 89$

A simple example $\quad 89$

A more complex example $\quad 90$

The keypad in SPSS $\quad 91$

SPSS exercises for selecting cases $\quad 92$

Subsets of variables $\quad 92$

Splitting files $\quad 93$

$\begin{array}{ll}\text { Weighting } & 93\end{array}$

An example of weighting $\quad 94$

Data manipulation $\quad 95$

Altering individual codes or groups of codes (Recode) 96

$\begin{array}{ll}\text { Combining codes } & 96\end{array}$

Example 1 96

Example 2 99

$\begin{array}{ll}\text { SPSS exercises for recoding variables } & 105\end{array}$

Arithmetical operations on a variable's codes (Compute) 105

$\begin{array}{ll}\text { SPSS exercises for Compute } & 107\end{array}$

If: using logical statements to create a new variable $\quad 107$

$\begin{array}{ll}\text { An example of using logical statements } & 107\end{array}$

Logical operators $\quad 110$

$\begin{array}{ll}\text { SPSS exercises for IF } & 112\end{array}$

$\begin{array}{ll}\text { Transformations using Count } & 112\end{array}$

SPSS exercise for Count $\quad 113$

New variables $\quad 114$

Labelling $\quad 114$

Missing values $\quad 114$

$\begin{array}{ll}\text { A final bit of advice about data manipulation } & 114\end{array}$

4 Hypothesis-Testing and $\boldsymbol{t}$-Tests $\quad 116$

Confirmatory statistics $\quad 116$

$\begin{array}{ll}\text { Hypothesis-testing } & 116\end{array}$

$\begin{array}{ll}\text { Statistical significance } & 118\end{array}$

Confirmatory statistics: $t$-tests $\quad 119$

Independent-Samples t-test: example 1 120

Independent-Samples t-test: example $2 \quad 123$

$\begin{array}{ll}\text { Paired-samples } t \text {-test (for dependent/matched groups) } & 124\end{array}$

Running the paired-sample t-test: an example 124

SPSS $t$-test exercise $\quad 126$

5 Crosstabulation $\quad 127$

$\begin{array}{ll}\text { Introduction } & 127\end{array}$

$\begin{array}{lr}\text { Crosstabs in SPSS } & 127\end{array}$ 
$\begin{array}{ll}\text { The Chi-square test } & 130\end{array}$

Measures of association $\quad 133$

A note of caution $\quad 134$

$\begin{array}{ll}\text { Chi-square: a second example } & 135\end{array}$

$\begin{array}{ll}\text { Introducing a control variable } & 138\end{array}$

Appendix: Measures of association $\quad 143$

$\begin{array}{ll}\text { Measures of association for nominal variables } & 143\end{array}$

$\begin{array}{ll}\text { Measures of association for ordinal variables } & 143\end{array}$

$\begin{array}{lr}\text { SPSS exercises on crosstabulation } & 144\end{array}$

6 Analysis of Variance (ANOVA) $\quad 145$

$\begin{array}{ll}\text { Introduction } & 145\end{array}$

How to do a simple ANOVA using SPSS $\quad 146$

ANOVA exercise $\quad 150$

Two-way analysis of variance (ANOVA) $\quad 150$

$\begin{array}{ll}\text { A two-way ANOVA for SPSS } & 150\end{array}$

$\begin{array}{ll}\text { Two-way ANOVA exercises } & 154\end{array}$

7 Correlation and Regression $\quad 155$

$\begin{array}{ll}\text { Scattergrams } & 155\end{array}$

$\begin{array}{ll}\text { Producing scattergrams with SPSS } & 157\end{array}$

$\begin{array}{ll}\text { Points about scattergrams } & 158\end{array}$

$\begin{array}{ll}\text { Scattergram exercise } & 159\end{array}$

$\begin{array}{ll}\text { Pearson's product-moment correlation coefficient }(r) & 160\end{array}$

$\begin{array}{ll}\text { Assumptions of the correlation coefficient } & 160\end{array}$

$\begin{array}{ll}\text { Correlating with SPSS } & 161\end{array}$

$\begin{array}{ll}\text { Interpretation of the correlation coefficient }(r) & 163\end{array}$

$\begin{array}{ll}\text { Exercises in producing correlation with SPSS } & 164\end{array}$

$\begin{array}{ll}\text { A final point on the correlation coefficient } & 165\end{array}$

$\begin{array}{ll}\text { Regression } & 165\end{array}$

$\begin{array}{ll}\text { Using SPSS to carry out a simple regression } & 165\end{array}$

$\begin{array}{ll}\text { Multiple regression } & 168\end{array}$

$\begin{array}{ll}\text { An SPSS example of multiple regression } & 169\end{array}$

$\begin{array}{ll}\text { Other considerations } & 171\end{array}$

$\begin{array}{ll}\text { Regression exercises } & 173\end{array}$

8 Factor Analysis $\quad 174$

$\begin{array}{ll}\text { Introduction } & 174\end{array}$

$\begin{array}{ll}\text { Using factor analysis in SPSS } & 175\end{array}$

$\begin{array}{ll}\text { Extraction } & 175\end{array}$

$\begin{array}{ll}\text { Rotation } & 179\end{array}$

$\begin{array}{ll}\text { Other considerations } & 183\end{array}$

$\begin{array}{ll}\text { Factor analysis exercises } & 185\end{array}$

9 Loglinear Analysis $\quad 186$

$\begin{array}{ll}\text { Introduction } & 186\end{array}$

$\begin{array}{ll}\text { Problems that loglinear analysis can answer } & 186\end{array}$

$\begin{array}{ll}\text { The 'logic' of loglinear analysis } & 187\end{array}$ 
$\begin{array}{lr}\text { Loglinear analysis: specific examples with SPSS } & 189\end{array}$

'Model selection' 190

'General loglinear analysis' 196

$\begin{array}{ll}\text { 'Logit' analysis } & 204\end{array}$

Conclusion $\quad 213$

$\begin{array}{ll}\text { Loglinear analysis exercises } & 213\end{array}$

10 Multiple Response Sets 217

$\begin{array}{ll}\text { Introduction } & 217\end{array}$

Using Multiple Response $\quad 222$

Creating a multiple response set from a group of dichotomous variables $\quad 222$

$\begin{array}{ll}\text { Creating a multiple response set from a group of categorical variables } & 225\end{array}$

$\begin{array}{ll}\text { Tabulating and crosstabulating multiple response sets } & 227\end{array}$

$\begin{array}{ll}\text { Tabulations } & 227\end{array}$

$\begin{array}{ll}\text { Crosstabulations } & 228\end{array}$

Summary 231

$\begin{array}{ll}\text { Multiple response set exercises } & 233\end{array}$

'Geometric' coding $\quad 233$

An example of geometric coding $\quad 233$

Working with geometric codes $\quad 236$

Conclusion $\quad 238$

$\begin{array}{ll}\text { 'Geometric' coding exercises } & 238\end{array}$

$\begin{array}{ll}\text { Conclusion } & 239\end{array}$

$\begin{array}{ll}\text { Choosing the correct statistical test } & 239\end{array}$

$\begin{array}{ll}\text { A note of caution } & 242\end{array}$

References $\quad 244$

Appendix 1: The dataset variables quick look-up guides 245

Appendix 2: Scales $\quad 271$

Appendix 3: Questions used to generate variables used in the practice datasets 274

Index 331 


\section{LIST OF FIGURES}

I.1 The positivist paradigm $\quad 5$

O.1a Starting SPSS $\quad 13$

O.1b SPSS for Windows 'opening dialog box'

$\begin{array}{ll}\text { O.2 Blank data Editor window (Data View) } & 15\end{array}$

$\begin{array}{lll}\text { O.3 Edit drop-down menu } & 17\end{array}$

O.4 Edit Options: General dialog box $\quad 18$

O.5 Édit Options: Output Labels dialog box 19

$\begin{array}{ll}\text { O.6 Opening a data file } & 20\end{array}$

$\begin{array}{lll}\text { O.7 Open file dialog box } & 20\end{array}$

O.8 Data Editor window 21

O.9 Variables dialog box $\quad 22$

O.10 Data Editor window with Value Labels displayed 23

O.11 Viewer window $\quad 24$

$\begin{array}{ll}\text { O.12 Saving a data file } & 25\end{array}$

O.13 Save Data As dialog box 26

$\begin{array}{ll}\text { O.14 Open File dialog box } & 27\end{array}$

$\begin{array}{lll}\text { O.15 Save output prompt } & 27\end{array}$

$\begin{array}{lll}\text { O.16 Print dialog box } & 28\end{array}$

1.1 Responses to the 'Drinking questionnaire' in grid format 31

1.2a Importing an SPSS portable file (BSACrime) using File Open Data 32

1.2b Saving an imported SPSS portable file as an SPSS *- sav. file - 33

1.2c Importing files from other spreadsheets using File Open Data 34

1.2d Importing spreadsheet files from other applications using Database Wizard 35

1.2e Text Import Wizard $\quad 36$

$\begin{array}{ll}\text { 1.2f Dialog box to create a new data file } & 37\end{array}$

1.2g Data entry directly to Data View window of the Data Editor 37

1.2h Questionnaire data entered directly to the Data View window of the 38

1.3a Deleting a case $\quad 39$

1.3b Deleting a variable $\quad 39$

1.3c Save Data dialog box 40

1.4a Opening Variable View in the Data Editor 45

1.4b Variable View window of the Data Editor 46

1.4c Editing/changing the Variable View window of the Data Editor 46

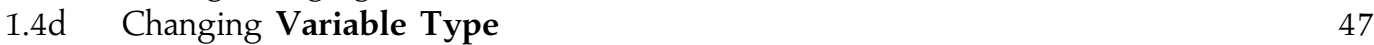

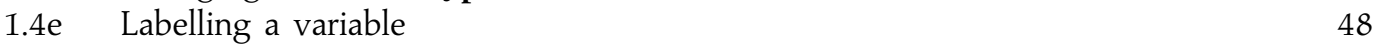

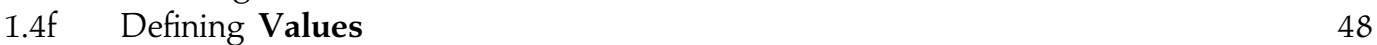

1.4g Defining Missing Values $\quad 49$

1.5a Drink survey data in Value format $\quad 49$

1.5b Drink survey data in Label format $\quad 50$

A.Ia Define Variable window $\quad 56$

A.Ib $\quad$ Define Labels window $\quad 57$ 
$\begin{array}{lll}\text { A.Ic Defining Missing Values } & 57\end{array}$

A.Id Defining the variable Type $\quad 58$

2.1 Descriptive Statistics and Frequencies $\quad 61$

2.2a Frequencies dialog box - 61

$\begin{array}{lll}2.2 \mathrm{~b} & \text { Frequencies dialog box } & 62\end{array}$

$\begin{array}{lll}2.3 & \text { Frequency table for marstat2 } & 63\end{array}$

$\begin{array}{lll}2.4 & \text { Frequency table for burglary } & 63\end{array}$

2.5 Statistics, Charts and Format options $\quad 66$

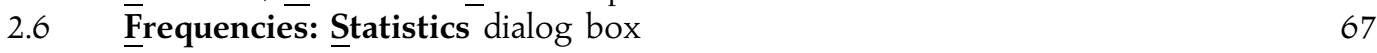

$\begin{array}{lll}2.7 & \text { Frequencies: } \text { Charts dialog box } & 67\end{array}$

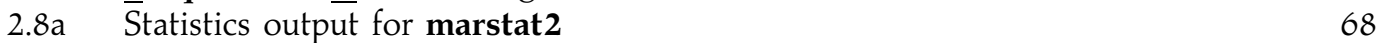

$\begin{array}{lll}2.8 \mathrm{~b} & \text { Bar chart for marstat2 } & 68\end{array}$

$\begin{array}{lll}2.9 & \text { Display frequency tables check box } & 69\end{array}$

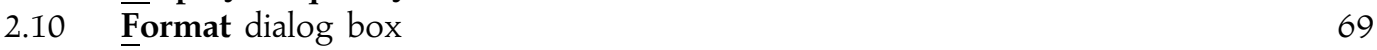

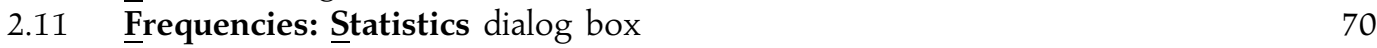

2.12a Descriptive statistics for rage $\quad 71$

$2.12 b$ Histogram for rage $\quad 71$

$\begin{array}{ll}2.13 & \text { Descriptive Statistics and Explore }\end{array}$

$\begin{array}{ll}2.14 & \text { Explore dialog box }\end{array}$

$\begin{array}{lll}2.15 & \text { Explore: } \text { Statistics dialog box } & 73\end{array}$

2.16a Descriptive statistics from Explore procedure $\quad 74$

2.16b Extreme values from Explore procedure $\quad 75$

2.16c Stem and leaf plots from Explore procedure $\quad 76$

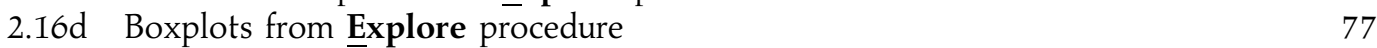

$\begin{array}{lll}2.17 & \text { Graphs drop-down menu } & 78\end{array}$

$\begin{array}{lll}2.18 & \text { Pie Charts dialog box } & 78\end{array}$

2.19 Define Pie: Summaries for Groups of Cases dialog box 79

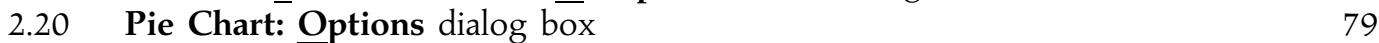

2.21 Pie chart for marstat2 $\quad 80$

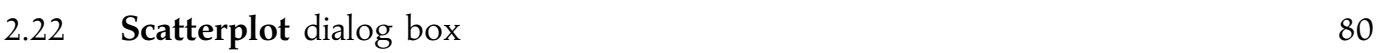

2.23 Simple Scatterplot dialog box $\quad 81$

$2.24 \quad$ Scatterplot of percap1 by rstatus $\quad 81$

$2.25 \quad$ Line Charts dialog box $\quad 82$

2.26 Define Simple Line: Summaries for Groups of Cases dialog box 83

2.27 Options dialog box $\quad-\quad 83$

$2.28 \quad$ Simple line chart for rearn $\quad 84$

2.29 Define Multiple Line: Summaries for Groups of Cases dialog box 85

$2.30 \quad$ Multiple line chart for rearn by rsex $\quad 85$

2.31 Chart Editor window $\quad 86$

2.32 Chart Editor window with Line Styles dialog box $\quad 87$

$\begin{array}{lll}3.1 & \text { Select Cases window } & 89\end{array}$

$\begin{array}{lll}3.2 & \text { Select } \overline{\text { If }} \text { subwindow } & 90\end{array}$

3.3 Example of selected cases on the Data View window 90

3.4 Example of a more complex selection using Select If 91

3.5 Example of splitting a file by rsex $\quad 93$

3.6 Choosing the Weight Cases window 94

3.7 Example of a variable, wtfactor, being used to weight a dataset 95

$\begin{array}{lll}3.8 & \text { Recoding age in years to Age categories } & 97\end{array}$

3.9a An example of Recode: rage into a new variable, agecats 98

$\begin{array}{lll}3.9 \mathrm{~b} & \text { Identifying old and new values for agecats } & 98\end{array}$ 
3.10 Recoding religion into a new variable: Old Categories

(from religion variable) and New Categories (relcats variable)

3.11a Collapsing the religion categories into a new variable, relcats

100

$3.11 \mathrm{~b}$ Identifying old and new variables for the new religion variable, relcats 100

3.12 Defining labels for the new variable, relcats, in the Variable View window 101

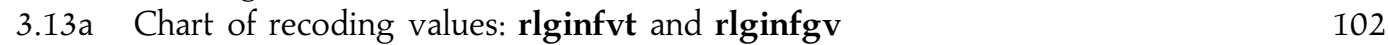

$3.13 \mathrm{~b}$ Recoding two variables into two new variables: rlginfvt and rlginfgv $\quad 102$

$3.13 \mathrm{C}$ Recoding new variables: relinfv2 and relinfg2 103

$\begin{array}{lll}3.14 & \text { Recoding chart } & 103\end{array}$

3.15a Example of using Automatic Recode to create a new variable $\quad 104$

$3.15 \mathrm{~b}$ Table displaying new and old values of the recoded variable $\quad 104$

3.16a Example of using Compute to create a new variable 106

3.16b A more complex example of using Compute to create a new variable $\quad 106$

3.17a Example of creating a new variable using Compute and If, first code 108

3.17b Example of Compute Variables using If: specifying the condition, first code 108

3.17c Example of creating a new variable using Compute and If, second code $\quad 109$

3.17d Example of Compute Variables using If: specifying the condition, 109 second code

3.17e Frequency count of the new variable, elite 110

3.18 Crosstabulation of rghclass by hedqual as a check on If statements 111

3.19a Main window for Count $\quad-\quad 113$

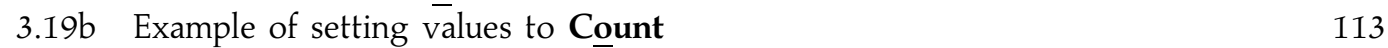

3.19c Frequency table of the newly created variable, cominv $\quad 114$

4.1a Descriptive statistics for number of cigarettes smoked per day (smokday) 116

4.1b Boxplot of number of cigarettes smoked per day (smokday) by sex (rsex) 117

$\begin{array}{lll}4.2 & \text { Errors in confirmatory statistics } & 118\end{array}$

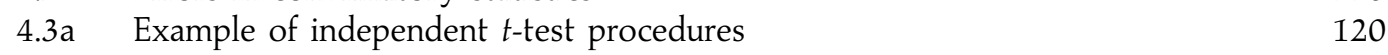

$4.3 \mathrm{~b} \quad$ Running the independent $t$-test $\quad 121$

4.3c Defining Values of Grouping Variable $\quad 121$

4.4 Independent $t$-test output: cigarettes smoked per day (smokday) by $\quad 122$ sex (rsex)

4.5 Independent $t$-test output: perceived crime (crime) by gender (rsex) 123

$\begin{array}{ll}4.6 \mathrm{a} & \text { Running the paired samples } t \text {-test } \\ 4.6 \mathrm{~b} & 125\end{array}$

$4.6 \mathrm{~b}$ Selecting the variables for the paired samples $t$-test $\quad 125$

$\begin{array}{ll}4.7 & \text { Output from the paired samples t-test } \\ 5.126\end{array}$

$\begin{array}{lll}5.1 & \text { Frequency table of soctrust } & 127\end{array}$

$\begin{array}{ll}\text { 5.2 Accessing the Crosstabs procedure } & 128\end{array}$

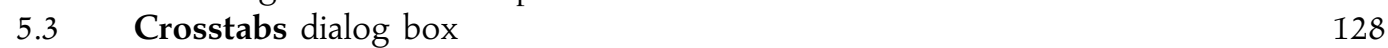

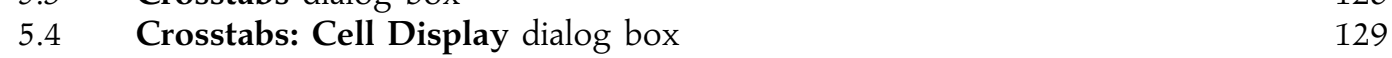

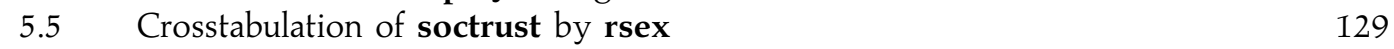

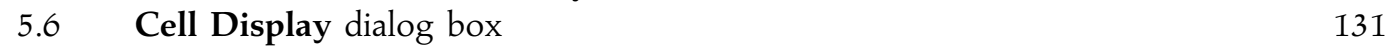

$\begin{array}{lll}5.7 & \text { Crosstabs: Statistics dialog box } & 131\end{array}$

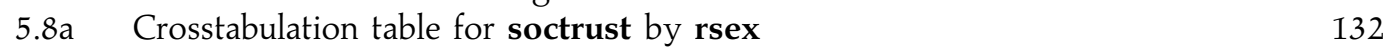

$\begin{array}{lll}5.8 \mathrm{~b} & \text { Chi-square results for soctrust by rsex } & 133\end{array}$

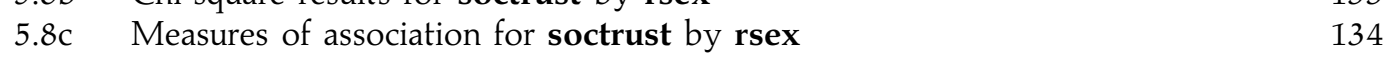

$\begin{array}{ll}5.9 & \text { Frequency table for homosex } \\ 5.10 & 135\end{array}$

5.10 Crosstabs: Cell Display dialog box 136

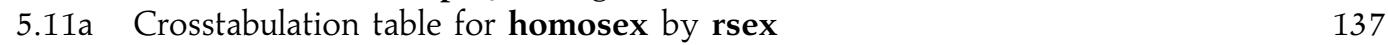

$\begin{array}{lll}5.11 \mathrm{~b} & \text { Chi-square results for homosex by rsex } & 137\end{array}$ 


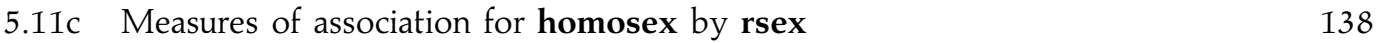

$\begin{array}{lll}5.12 & \text { Frequency table for newage } & 138\end{array}$

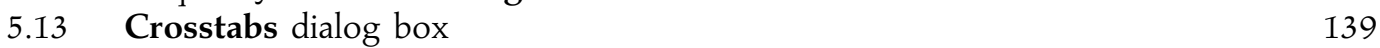

5.14a Crosstabulation table for homosex by rsex by newage 140

$5.14 \mathrm{~b}$ Chi-square results for homosex by rsex by newage 141

5.14c Measures of association for homosex by rsex by newage 142

6.1 Diagram depicting a significant and nonsignificant ANOVA result 146

$\begin{array}{lll}6.2 & \text { One-Way ANOVA window } & 147\end{array}$

6.3 One-Way ANOVA: Options window $\quad 147$

$6.4 \quad$ One-Way ANOVA output $\quad 148$

6.5 One-Way ANOVA: Post-Hoc Multiple Comparisons window 149

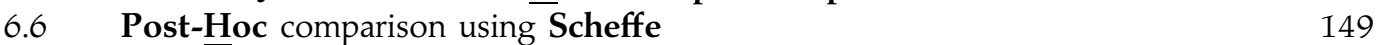

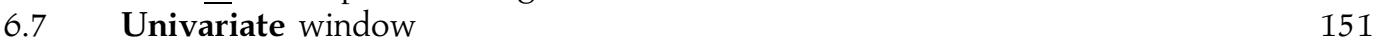

6.8 Univariate Analysis of Variance $\quad 152$

6.9 Univariate: Profile Plots $\quad 153$

6.10 Profile Plots $\quad 154$

7.1a A positive relationship between two variables 156

7.1b A negative relationship between two variables $\quad 156$

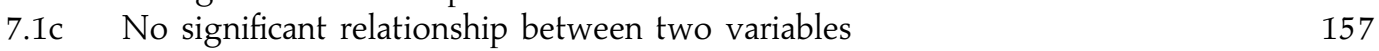

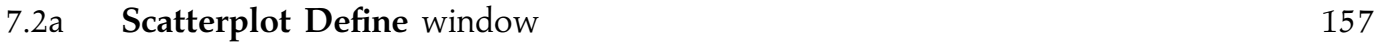

$\begin{array}{lll}7.2 \mathrm{~b} & \text { Simple Scatterplot window } & 158\end{array}$

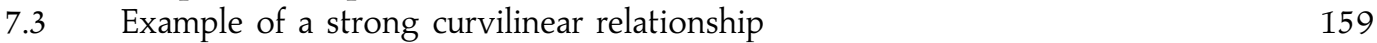

$\begin{array}{lll}7.4 & \text { Bivariate Correlations window } & 161\end{array}$

$\begin{array}{lll}\text { 7.5 Pearson product-moment correlation coefficient between respondents' } & 162\end{array}$

perception of crime, TV watched during the week and TV watched at weekends

$\begin{array}{lll}\text { 7.6 } & \text { Linear Regression window } & 166\end{array}$

$\begin{array}{lll}7.7 & \text { Regression results } & 166\end{array}$

$\begin{array}{lll}7.8 & \text { How variables may overlap } & 168\end{array}$

$\begin{array}{ll}7.9 & \text { Linear Regression window } \\ 7.10 & 170\end{array}$

$\begin{array}{lll}7.10 & \text { Multiple Regression results } & 171\end{array}$

$\begin{array}{lll}7.11 & \text { Linear Regression: Options subwindow } & 173\end{array}$

8.1 Simple example of what Factor Analysis does $\quad 174$

$\begin{array}{ll}\text { 8.2 Correlation matrix between all the variables } & 176\end{array}$

$\begin{array}{ll}8.3 & \text { Factor Analysis main window } \\ 8.476\end{array}$

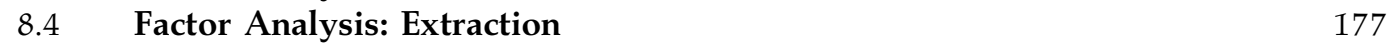

$\begin{array}{lll}8.5 & \text { Factor Analysis output } & 177\end{array}$

8.6a Factor Analysis main window: 'rotation example' 180

8.6b Factor Analysis: Rotation subwindow 181

$\begin{array}{lll}8.7 & \text { Factor Analysis } & 181\end{array}$

9.1 A by B, 'chance' and 'actual' distributions 188

9.2a A by B for two levels of C, 'three-way' interaction absent 188

9.2b A by B for two levels of C, 'three-way' interaction present 188

$\begin{array}{lll}9.3 & \text { Selecting Loglinear } & 191\end{array}$

$\begin{array}{ll}\text { 9.4a The Model Selection window } & 192\end{array}$

\begin{tabular}{ll}
$9.4 \mathrm{~b}$ & The Define Range subwindow \\
\hline $9.4 \mathrm{c}$
\end{tabular}

$\begin{array}{ll}\text { 9.4c The Model subwindow } & 193\end{array}$

9.4d The Options subwindow $\quad 193$

9.5 Results for a 'backward elimination' loglinear analysis $\quad 194$

$\begin{array}{ll}\text { 9.6a General loglinear analysis } & 197\end{array}$ 
9.6b General loglinear analysis model of two-way interactions $\quad 197$

9.6c The Options subwindow $\quad 198$

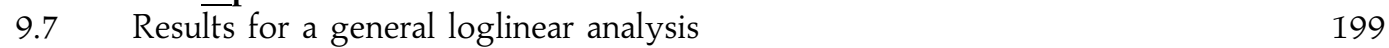

9.8a General loglinear analysis with rage included as a covariate 203

$9.8 \mathrm{~b}$ Model for a general loglinear analysis model of two-way interactions $\quad 204$ with rage included

9.9 Results for a general loglinear analysis with rage included as a covariate 205

$\begin{array}{lll}9.10 a & \text { Logit main window } & 208\end{array}$

9.10b Logit Model with majors included $\quad 209$

9.10c Logit Options subwindow 209

9.11 Results for a Logit analysis with majors included $\quad 210$

9.12 Logit Model with majors excluded $\quad 212$

9.13 Results for a Logit analysis with majors excluded $\quad 214$

10.1a Normal Frequencies counts of the seven abortion variables 218

10.1b Normal Frequencies counts of club variables 220

10.1c Normal Frequencies counts of bprior1 and bprior2 221

10.2 Selecting Multiple Response from the Analyze menue 223

10.3a Define Multiple Response Sets window for a group of dichotomous 224 variables

10.3b Frequency count for a dichotomous response set (\$mrabort) 224

10.4a Define Multiple Response Sets window for a group of category variables, $\quad 225$ each with a unique code

10.4b Define Multiple Response Sets window for a group of category variables, 226 each with the same code

10.4c Frequency counts for categorical multiple response sets (\$mrclubs) 227 and (\$mrprior)

10.5 Window for obtaining a frequency count of Multiple Response variables 228

10.6a Multiple Response Crosstabs window 228

10.6b Multiple Response Crosstabs: Define Variable subwindow 229

10.6c Multiple Response Crosstabs: Option subwindow with column 229 percentages based upon the number of responses

10.7 Crosstabulation of a multiple response set (mrabort) by a categorical 230 variable (recrelig), column percentages with percentages and totals based upon the number of responses

10.8 Multiple Response Crosstabs: Option subwindow with row percentages based upon the number of cases

10.9 Crosstabulation of a multiple response set (mrabort) by a categorical variable (recrelig), row percentages with percentages and totals based upon the number of cases

10.10 Frequency output for a 'geometric' variable geoabort 235

10.11 Crosstabulation of the recoded 'geometric' anti-abortion variable 237 (recgeoab) by religion (recrelig-Catholic or All others)

C.1 The triangular relationship between 'research problem', data and statistical 240 procedures

C.2 Choosing the correct statistical procedure 


\section{PREFACE}

Statistics and quantitative methods courses in the social sciences often suffer from an inability to make a link between the skills they seek to impart and present-day society. They can be made more attractive to students by illustrating analytic methods with examples from the contemporary world and involving students in computer analyses of real data. As teachers of statistics and quantitative methods courses in three universities in the British Isles, we were aware of the need for locally interesting datasets that would be available to students in the social sciences. The availability of the British Social Attitudes (BSA) Survey brought with it the possibility of utilising the research data that had been collected in teaching. ${ }^{1}$ This use is particularly appropriate given the commitment of the organisers of the BSA surveys to disseminating the results of the survey to the widest possible audiences.

As well as providing 'raw material' for statistical analysis exercises on a research methods training course, the four datasets - 'Crime', 'Health', 'Welfare' and 'Politics' - constitute significant bodies of information about contemporary British society. The data provide the basis for the substantive consideration of attitudes and social structure in Britain and could be used to great effect on social science courses in disciplines such as Criminology, Health Studies, Sociology, Social Policy and Political Science.

\section{Layout and scope of the book}

The text begins with two introductory chapters. The 'Introduction' chapter places 'the quantitative perspective' within the landscape of the social sciences and then moves on to discuss the logic underlying statistical analysis. This is followed by an 'Orientation' chapter that gives information about the British Social Attitudes Survey and explains the Windows 'environment' as it relates to SPSS. This chapter tells the student about the general layout of SPSS and gives advice about general 'housekeeping' that will ensure that carrying out practical work with the program is efficient and trouble-free. SPSS has built-in features for advising and helping users. How to access and use these is explained in this 'Orientation' chapter.

The two introductory chapters are followed by ten modules that provide instruction about the practicalities of carrying out statistical analyses with SPSS. This begins with Module 1 on 'Data Input', moves through procedures for looking at individual variables in Module 2, 'Listing and Exploring Data', to the important topic of data refinement in Module 3, 'Data Selection and Manipulation', and then on to seven modules that present the practicalities of different types of statistical analysis with SPSS. The text ends with a 'Conclusion' chapter that provides advice about the procedures for selecting appropriate statistical tests.

\footnotetext{
${ }^{1}$ Note that the four datasets have been adapted from the BSA for use as teaching datasets. While the data are of high quality, changes have been made to make them more suitable for student use - most notably the simplification of the missing values codes used in the survey and the construction of additional scales for teaching purposes. This different treatment of missing values means that some of the percentage tabulations given in Appendix 3 may not agree precisely with those found in the teaching datasets. Academics wishing to use the BSA data for research purposes must make use of the original BSA datasets.
} 
The four practice datasets are integral to the successful use of this textbook and it is essential that students have a genuine understanding of the contents of these datasets. To make this understanding possible, three Appendixes are provided:

- Appendix 1 - The Dataset Variables Quick Look-up Guides. A comprehensive listing and brief description of all the variables in the datasets so that students can locate the variables they need when carrying out exercises.

- Appendix 2 - Scales. Descriptions of each of the scales contained in the datasets, including details of their meaning and construction.

- Appendix 3 - Questions Used to Generate Variables Used in the Practice Datasets. A reproduction of the exact wordings and response options used in the actual questions asked by the British Social Attitudes interviewers are given so that students can have a genuine understanding of the meaning of the variables they are using in their analyses.

Finally, the text is intended as an introduction to the main data features of SPSS and provides careful step-by-step instruction in the practical details of carrying out statistical procedures and the interpretation of SPSS output. While this necessarily requires the discussion of the logic underlying many of the statistical procedures, this book is not intended to be a 'stand-alone' statistical text. It should be used on a course of study in conjunction with a statistics textbook and/or a program of lectures and readings provided by the instructor. 


\section{ACKNOWLEDGEMENTS}

The organisers of the British Social Attitudes Survey located in the National Centre for Social Research have overall responsibility for the BSA series. We are grateful for their support and interest in the development of the textbook and that they made the data available for the construction of the practice datasets that accompany this text and allowed us to duplicate portions of the BSA interview schedule and questionnaires.

SPSS, Inc. has allowed us to reproduce windows and output generated by the SPSS programme. This obviously was essential for this text and we are most appreciative of their permission.

Finally, the students at the Queen's University, Belfast, the University of Ulster and Sheffield Hallam University who used a draft version of the workbook during the academic year 19992000 provided essential feedback that allowed us to identify areas where the text could be improved. Again, we are grateful for their tolerance and good humour. 